Trauma Berufskrankh 2015 - [Suppl 2]:

17:293-300

DOI 10.1007/s10039-015-0021-9

Online publiziert: 25. Juni 2015

(c) Springer-Verlag Berlin Heidelberg 2015

CrossMark

Wolf-Dieter Müller

Abteilung Orthopädie/Unfallchirurgie, m\&i Fachklinik Bad Liebenstein, Bad Liebenstein, Deutschland

\title{
Das Reha-Management der DGUV
}

\section{Erfahrungen und Erwartungen aus der Praxis einer Rehaklinik}

\begin{tabular}{|ll}
\hline SF-36 & $\begin{array}{l}\text { Short Form - 36 Health } \\
\text { Servey }\end{array}$ \\
\hline UV-Träger & Unfallversicherungsträger \\
\hline VAV & Verletztenartenverfahren \\
\hline VDR & $\begin{array}{l}\text { Verband Deutscher Renten- } \\
\text { versicherungsträger }\end{array}$ \\
\hline WOMAC & $\begin{array}{l}\text { Western Ontario and } \\
\text { McMaster Universities } \\
\text { Osteoarthritis Index }\end{array}$ \\
\hline
\end{tabular}

Das einzelfallbezogene Reha-Management ist seit Jahren ein fester und unverzichtbarer Bestandteil in der Heilverfahrenssteuerung der DGUV (Deutsche Gesetzliche Unfallversicherung). Die bisherigen Erfahrungen der einzelnen Unfallversicherungsträger im Reha-Management wurden in einem EckpunktePapier 2008 sowie konkretisiert in einem Handlungsleitfaden 2010 zusammengefasst und 2014 aktualisiert [1, 2]. Dabei war es das Ziel, ein einheitliches Bild bezüglich des Reha-Managements der Gesetzlichen Unfallversicherung gegenüber Versicherten, Unternehmern und anderen beteiligten Rehabilitationspartnern zu fördern und den Unfallversicherungsträgern (UV-Trägern) gleichzeitig zu ermöglichen, branchen- und aufgabenbezogene Besonderheiten zu erhalten [2]. Im Rahmen dieses dynamischen Prozesses bringen Rehabilitationskliniken mit Beteiligung an der Berufsgenossenschaftlichen Stationären Weiterbehandlung (BGSW) wesentliche Kompetenzen ein. In dem nachfolgenden Beitrag sollen langjährige Erfahrungen einer BGSW-Klinik mit dem Reha-Management der DGUV und die daraus resultierenden Erwartungen an dessen zukünftige Weiterentwicklung dargestellt werden.

\section{Rolle der BGSW-Klinik im Reha-Management der DGUV}

Rehabilitations- bzw. BGSW-Kliniken können als Netzwerkpartner im Heilverfahren der DGUV gemeinsam mit Durchgangsärzten (D-Ärzten) und Reha-Managern eine Schrittmacher- und Lotsenfunktion übernehmen. Diese besondere Rolle resultiert zunächst aus der Tatsache, dass die im Handlungsleitfaden geforderte Orientierung des RehaManagements an dem biopsychosozialen Modell der internationalen Klassifikation der Funktionsfähigkeit Behinderung und Gesundheit (ICF) seit Jahren als obligatorische konzeptionelle Basis der Arbeit in modernen zertifizierten BGSW-Kliniken gilt. Zudem ist in keinem anderen Abschnitt des Heilverfahrens eine derart direkte kontinuierliche Arbeit mit den Rehabilitanden über längere Zeiträume gegeben wie während der stationären Rehabilitation. In der BGSW-Klinik besteht ein ganztägiger Kontakt zwischen Reha-Team und Rehabilitanden über Wochen, was beim Aufdecken heilverfahrensrelevanter Kontextfaktoren eine entscheidende Hilfe sein kann. Die besondere Rolle der BGSW-Kliniken begründete sich auch in der Tatsache, dass mit Ausnahme der Komplexen Stationären Rehabilitation (KSR), die BG-Kliniken vorbehalten ist, je nach Spezialisierungsgrad das vollständige rehabilitative Leistungsspektrum der DGUV mit Frührehabilitation, Berufsgenossenschaftlicher Stationärer Weiterbehandlung (BGSW), Erweiterter Ambulanter Physiotherapie (EAP), Arbeitsplatzbezogener Muskuloskeletaler Rehabilitation (ABMR) sowie ambulanten Leistungen (Physiotherapie, Ergotherapie, Testverfahren) angeboten wer- 
den kann. Überdies ist in BGSW-Kliniken die permanente Präsenz eines interdisziplinären Reha-Teams mit umfassenden Möglichkeiten rehabilitativer Diagnostik und Therapie abgesichert. Schließlich resultiert die Schrittmacher- und Lotsenrolle der BGSW-Klinik auch aus der Tatsache, dass BGSW-Patienten meistens auch Verletzungen erlitten haben, die zum Reha-Management der UV-Träger gehören. Das Beispiel einer eigenen Stichprobe (397 BGSW-Patienten eines Netzwerkpartners aus den Jahren 2010 und 2011) zeigte, dass die durchschnittliche Gesamtdauer der Arbeitsunfähigkeit dieser Rehabilitanden knapp 177 Tage betrug und somit weit über der ein Reha-Management begründenden 112-Tage-Grenze des Handlungsleitfadens der DGUV lag.

Alle genannten Argumente zur Bedeutung und positiven Rollenfunktion von BGSW-Kliniken im DGUV-RehaManagement kommen allerdings nur dann zum Tragen, wenn es in naher $\mathrm{Zu}$ kunft gelingt, diese Einrichtungen fest in die neugestalteten 3-stufigen Traumanetzwerkstrukturen einzugliedern und ein „Trauma-Reha-Netzwerk“ zu entwickeln. Ein entsprechender Lösungsansatz wird in diesem Beitrag vorgestellt.

\section{Eigene Erfahrungen zur Entwicklung und Optimierung des Reha-Managements der DGUV}

Mit der Beteiligung unserer Rehabilitationsklinik am BGSW-Verfahren der DGUV ergab sich für unser Haus eine Reihe neuer Anforderungen, denen durch eine entsprechende Anpassung und Optimierung des Rehabilitationskonzepts und des klinikinternen Rehabilitationsmanagements schnell Rechnung getragen werden musste. Dabei zeigte sich in der Anfangsphase unserer Zusammenarbeit mit den Unfallversicherungsträgern Ende der 90er Jahre, dass sowohl auf dem Gebiet des branchenspezifischen Reha-Managements als auch hinsichtlich der geforderten Qualitäts-, Effektivitäts- und Effizienznachweise teilweise unterschiedliche Anforderungen seitens der einzelnen UV-Träger an die BGSW-Klinik gestellt wurden.
Obwohl unser Haus zum damaligen Zeitpunkt die zur BGSW-Beteiligung notwendigen Anforderungen an Struktur- und Prozessqualität erfüllte, gab es zweifellos Defizite hinsichtlich objektiver Nachweise der erreichten Ergebnisqualität durchgeführter Heilverfahren. Aus diesem Grund setzten wir uns das Ziel, ein Qualitätssicherungssystem zu entwickeln, das auf der Basis eines multidimensionalen Assessments eine Heilverfahrenssteuerung erlaubte und die kontinuierliche Optimierung von Struktur-, Prozess- und Ergebnisqualität des BGSWVerfahrens anhand der Ergebnisse dieses Rehabilitationsassessments innerhalb kurzer Zeit ermöglichte. Beteiligte Kooperationspartner bei diesem Projekt waren die Verwaltungsberufsgenossenschaft (VBG) und das Institut für Physiotherapie der Friedrich-Schiller-Universität Jena. In der Zeit von 2002 bis 2008 erprobten und implementierten wir dieses System in das Reha-Konzept unserer Klinik. Auf dieser Grundlage erreichten wir eine schrittweise Optimierung des BGSWVerfahrens und arbeiteten an der Entwicklung des ABMR-Verfahrens mit [3]. Am Ende dieses Entwicklungsprozesses kristallisierten sich die folgenden 4 Kernelemente als Erfolgsfaktoren für ein wirksames Reha-Management heraus:

- der Einsatz patientenbezogener Assessmentinstrumente (Patient Reported Outcomes, PROs),

- die Nutzung arbeits(platz)bezogener

Test- und Therapieelemente,

- die Durchführung regelhafter Fallkonferenzen und

- jährliche Reviews hinsichtlich der geleisteten Arbeit und der erreichten Ergebnisse [4].

\section{Einsatz patientenbezogener Assessmentinstrumente}

Die Auswahl geeigneter Assessmentinstrumente wurde nach einem klar definierten Kriterienkatalog vorgenommen. Es sollte die Erfassung des allgemeinen und des spezifischen Gesundheitszustandes sowie der Lebensqualität erfolgen. Die Instrumente sollten praxistauglich sein und in ihrer Anwendung einen geringstmöglichen personellen, bürokratischen sowie finanziellen Mehraufwand erfordern (ausschließlicher Einsatz selbstadministrierter Patientenfragebogeninstrumente). Die Instrumente sollten zur Verfahrenssteuerung geeignet sein, d. h., eine schnelle Auswertung sollte eine simultane Nutzungsmöglichkeit während des BGSW-Verfahrens zulassen. Schließlich sollte es sich um anerkannte und bewährte Messinstrumente handeln, welche die üblichen Testgütekriterien hinsichtlich Objektivität, Reliabilität, Validität und Änderungssensitivität erfüllen. Auf der Basis dieses Kriterienkataloges und im Ergebnis einer Reihe von Voruntersuchungen wählten wir folgende Assessmentinstrumente aus und nutzen sie seit 2002 bis dato in Kooperation mit einigen UV-Trägern:

- allgemeine Gesundheit: SF-36 (Short Form - 36 Health Survey; [5]),

- spezifische Gesundheit obere Extremitäten: DASH (Disabilities of the Arm, Shoulder and Hand; [6]),

- spezifische Gesundheit Wirbelsäule: FFbH-R (Funktionsfragebogen Hannover - Rückenschmerzen; [7]),

- spezifische Gesundheit untere Extremitäten: WOMAC (Western Ontario and McMaster Universities Osteoarthritis Index; [8]),

- Lebensqualität: EQ-5D (European Quality of Live Questionaire; [9]).

Die Befragung der Rehabilitanden erfolgt zum Reha-Antritt, am Ende der dritten Behandlungswoche sowie im Follow-up 6 Monate nach Entlassung aus der BGSW. Bei Verlängerung des BGSW-Verfahrens wird eine zusätzliche Befragung am Ende der BGSW durchgeführt. Die Auswertung und Übermittlung der Messergebnisse wird „online“ durch assoziierte Kooperationspartner (Institut für Community Medicine, Ernst-Moritz-Arndt-Universität Greifswald sowie Institut für Physiotherapie der Friedrich-Schiller-Universität) gewährleistet, sodass die Daten spätestens 2 Tage nach der Befragung zur Verfügung stehen und zur Heilverfahrenssteuerung genutzt werden können. Für die Analyse und Bewertung der Assessmentergebnisse wurde ein Auswertungsalgorithmus entwickelt und den am Prozess beteiligten Reha-Managern und Ärzten durch entsprechende Schulungen vermittelt, sodass die Beurteilung 
und Nutzung der Daten nach einheitlichen und definierten Grundsätzen erfolgen kann.

Nach mittlerweile über 10-jährigem, regelhaftem Einsatz dieser patientennahen Assessment instrumente sind unsere Erfahrungen hinsichtlich des Nutzens für ein erfolgreiches Reha-Management durchweg positiv. Sie helfen bei der Aufdeckung von larvierten Kontextfaktoren, können für die individuelle Reha-Steuerung und Ableitung von Therapieschwerpunkten oder zur Abstimmung von Verlängerungsentscheidungen mit dem UVTräger genutzt werden und dienen der laufenden Effektivitätskontrolle sowie der stufenweisen Prozessoptimierung der Heilverfahren. Die Messergebnisse können Grundlage für Effektivitäts- bzw. Kosteneffizienzanalyseverfahren sein und gelten mittlerweile als ,einheitliche Währung “ zur Bewertung von Therapieverläufen und Reha-Ergebnissen für uns als Leistungserbringer und die beteiligten Leistungsträger. Das entscheidende Argument für den Einsatz dieser Assessmentinstrumente ist zweifellos der strenge Patientenbezug und die damit verbundene Anforderung an den Rehabilitanden, mit der Beantwortung der systematischen und standardisierten Fragenkataloge eine umfassende und strukturierte Selbsteinschätzung vorzunehmen und damit ein selbsterarbeitetes Problembewusstsein als solide Grundlage für einen effektiven und erfolgreichen Reha-Prozess zu entwickeln. Letztlich gibt der Umgang des Rehabilitanden mit den Fragebogeninstrumenten dem Reha-Team auch wichtige Hinweise hinsichtlich seiner Motivation und Compliance. Insofern ist der vom Rehabilitanden zu fordernde Aufwand in jedem Fall sinnvoll und akzeptabel.

\section{Arbeits(platz)bezug}

Die schnellstmögliche Wiedereingliederung der Unfallverletzten in den Arbeitsprozess ist die zentrale strategische Aufgabe des Reha-Managements der gesetzlichen Unfallversicherung. Das bedeutet, dass bereits zu Beginn der Rehabilitation der Fokus aller am Reha-Management beteiligten Akteure auf eventuelle aus den Verletzungsfolgen resultieren-

Trauma Berufskrankh 2015 · [Suppl 2]: 17:293-300 DOI 10.1007/s10039-015-0021-9

(c) Springer-Verlag Berlin Heidelberg 2015

\section{W.-D. Müller}

\section{Das Reha-Management der DGUV. Erfahrungen und Erwartungen aus der Praxis einer Rehaklinik}

\section{Zusammenfassung}

Rehabilitationskliniken mit Beteiligung an der Berufsgenossenschaftlichen Stationären Weiterbehandlung (BGSW) können als Netzwerkpartner im Heilverfahren der Deutschen Gesetzlichen Unfallversicherung (DGUV) gemeinsam mit Durchgangsärzten (D-Ärzten) und Reha-Managern eine Schrittmacher- und Lotsenfunktion übernehmen. Eigene Erfahrungen zur Entwicklung und Optimierung des Reha-Managements der DGUV zeigten, dass der Einsatz von Patient Reported Outcomes (PROs), arbeits(platz)bezogene Test- und Therapieelemente, die Durchführung regelhafter Fallkonferenzen und jährliche Reviews hinsichtlich der erreichten Ergebnisse wichtige Erfolgsfaktoren sind. Das durch die Versor- gungsstufenstruktur des neuen Heilverfahrens der DGUV geprägte Netzwerk zwischen Leistungsträgern und Leistungsanbietern sollte zu einem Trauma-Reha-Netzwerk mit integrierten Reha-Kliniken unterschiedlicher Versorgungsstufen weiterentwickelt werden. Ein vorgeschlagenes Phasenmodell Rehabilitation der DGUV könnte helfen, aktuell noch bestehende Verfahrenslücken zu schließen.

\section{Schlüsselwörter}

Stationäre Rehabilitation .

Berufsgenossenschaft · Arbeitsunfall ·

Phasenmodell Trauma-Rehabilitation . Heilverfahren

\section{Rehabilitation management of the DGUV. Experiences and expectations in the daily practice of a rehabilitation clinic}

\section{Abstract}

Rehabilitation and, especially employers liability insurance inpatient extended treatment (BGSW) facilities are predestined as network partners of traumatologists, rehabilitation managers and other groups for playing an important role as guides and pacemakers of the rehabilitation process. Our own experiences gained during developing and optimizing the rehabilitation management standards of the Association of German Statutory Accident Insurance (DGUV) institutions indicate that the systematic use of patient-reported outcomes, implementing workplacerelated diagnostic and therapeutic components in rehabilitation process, holding multiprofessional case conferences and undertak- ing regular reviews of current cases, could be identified as reliable predictors of success in rehabilitation after work-related injuries. The established, hierarchically organized trauma care networks could be supplemented by a network of rehabilitation facilities at all healthcare levels. The postulated phase model of trauma rehabilitation could be suitable for closing gaps in existing trauma treatment procedures.

\section{Keywords}

In-patient rehabilitation - Employers liability insurance association · Workrelated accident . Phased model of trauma rehabilitation - Medical treatment de Beeinträchtigungen der Teilhabe am Arbeitsleben gerichtet werden muss. In eigenen früheren Untersuchungen konnten wir zeigen, dass die Implementierung von arbeits(platz)bezogenen Rehabilitationskonzepten auf der Basis strukturierter Vergleiche von Arbeitsplatzanforderungs- und Patientenfähigkeitsprofilen Rehabilitationsergebnisse signifikant verbessern kann [10-12]. Daraus resultiert, dass in der BGSW-Klinik - unabhängig vom Spezialisierungsgrad - grundlegende arbeits(platz)bezogene Diagnostik- und Therapieverfahren in das Rehabilitationskonzept eingefügt werden müssen. Mit der Arbeitsplatzbezogenen Muskuloskeletalen Rehabilitation (ABMR) steht ein Heilverfahren der DGUV zur Verfügung, das punktgenau die Aspekte der arbeits(platz)bezogenen Test- und Trainingsmethoden berücksichtigt und im Reha-Management als weiteres Bindeglied zwischen medizinischer Rehabilitation und beruflicher Wiedereingliederung genutzt werden kann. 
Infobox 1 Erhebung des arbeits(platz)bezogenen Anforderungsprofils

\section{- Arbeitgeberbefragung \\ - Rehabilitandenbefragung \\ - Betriebsarztbefragung \\ - Arbeitsplatzbegehung \\ - Arbeitsplatzdatenbanken \\ - Assessment- und Profilvergleichsinst- rumente (z. B. IMBA - Integration von Menschen mit Behinderungen in die Arbeitswelt) [13]}

Infobox 2 Assessmentinstrumente zur Erfassung des arbeits(platz)bezogenen Fähigkeitsprofils

- PACT (Performance Assessment and Capacity Testing) nach Matheson

- FCE (Functional Capacity Evaluation)-Tests [z. B. EFL (Evaluation der funktionellen Leistungsfähigkeit) nach Isernhagen als Voll- oder Screening-Tests mit 5 bis 8 Testitems zu kritischen Arbeitsbelastungen, ERGOS, SAPPHIRE]

- IMBA (Integration von Menschen mit Behinderungen in die Arbeitswelt)

- PROs (Patient Reported Outcomes)

Für die praktische Umsetzung der beruflichen Teilhabeaspekte im Reha-Management der DGUV sind die nachfolgend aufgeführten Anforderungen zu erfüllen.

\section{Ermittlung des Arbeitsplatzanforderungsprofils}

Das exakte Arbeitsplatzanforderungsprofil stellt die Arbeits- und Orientierungsgrundlage für das Reha-Team in der BGSW-Klinik dar und muss zu Beginn der Rehabilitationsbehandlung vorliegen. Die zeitgerechte Bereitstellung des Arbeitsplatzanforderungsprofils ist eine Bringpflicht der Leistungsträger. Die verschiedenen Erhebungsmöglichkeiten sind in $\bullet$ Infobox 1 aufgezeigt.

Von den in der $\bullet$ Infobox 1 angeführten Erhebungsmethoden hat sich nach unseren praktischen Erfahrungen die direkte Arbeitgeberbefragung durch den verantwortlichen Reha-Manager als die verlässlichste Vorgehensweise bewährt. Die Dokumentation der Arbeitgeberauskunft muss in einem strukturierten Protokoll vorgenommen und dem Reha-Team der Klinik zu Beginn des dortigen Heilverfahrens zur Verfügung gestellt werden. Dabei ist es hilfreich, wenn zur Erfassung dieser arbeits(platz)bezogenen Anforderungsparameter ein standardisiertes Profilvergleichssystem genutzt und verfahrensübergreifend für EAP, BGSW und ABMR eingesetzt wird.

\section{Ermittlung des arbeits(platz)- bezogenen Fähigkeitsprofils}

Die Ermittlung des arbeits(platz)bezogenen Fähigkeitsprofils des Rehabilitanden ist eine notwendige Kernkompetenz des Reha-Teams der Rehabilitationseinrichtung. Die Erhebungsmethodik umfasst einerseits eine exakte und strukturierte Rehabilitandenbefragung unter Berücksichtigung des vorliegenden Arbeitsplatzanforderungsprofils. Andererseits müssen eine sorgfältige ärztliche Untersuchung und, wenn erforderlich, eine weiterführende klinische Diagnostik erfolgen, um alle verletzungsbedingten Struktur-, Funktions- und Aktivitätsstörungen genau zu erfassen. Danach werden die sog. kritischen Arbeitsbelastungen identifiziert, auf die der besondere Fokus der weiterführenden Rehabilitationsbehandlung gerichtet werden muss. Die während der Aufnahmeuntersuchung durch den Arzt oder Therapeuten erfassten Struktur- und Funktionsstörungen sowie klinisch-diagnostischen Befunde allein reichen für eine umfassende und exakte Beurteilung des arbeits(platz)bezogenen Fähigkeitsprofils des Patienten in vielen Fällen nicht aus. Hierbei werden vor allem ärztlicherseits die Unschärfen, die sich aus der rein struktur- und funktionsbasierten Beurteilung komplexer arbeitsbezogener Aktivitätsanforderungen ergeben, unterschätzt. Deshalb ist der Einsatz von Assessmentinstrumenten sinnvoll, die über den reinen struktur- und funktionsbezogenen Ansatz hinaus unter anderem auch definierte arbeits(platz)bezogene Aktivitätstests beinhalten. Damit wird erreicht, dass die Beurteilung der arbeits(platz)bezogenen Fähigkeiten eines Rehabilitanden nicht nur auf der durch medizinischen Sachverstand geprägten „Erfahrungsebene“ erfolgt, sondern auch eine objektive bzw. empirische Bewertungsebene hinzugefügt wird. Geeig- nete Assessmentinstrumente zur Überprüfung des arbeits(platz)bezogenen Fähigkeitsprofils sind in $\bullet$ Infobox $\mathbf{2}$ zusammengefasst.

\section{Profilvergleich}

Im Profilvergleich wird das ermittelte Patientenfähigkeitsprofil mit dem funktionalen Arbeitsplatzanforderungsprofil abgeglichen. Die dabei identifizierten körperlichen und funktionalen $\mathrm{Re}$ habilitationsdefizite stellen die Grundlage zur Therapieplanung und Rehabilitationszielvereinbarung mit dem Rehabilitanden dar. Zur anschaulichen und übersichtlichen Darstellung dieses Abgleiches ist die Anwendung etablierter Profilvergleichssysteme wie beispielsweise des Systems IMBA vorteilhaft.

\section{Arbeits(platz)bezogene Rehabilitationsinhalte}

Das verordnete Rehabilitationsprogramm soll gezielt auf die im Profilvergleich ermittelten Funktionalitätsdefizite abgestimmt werden. Voraussetzung dafür ist ein erreichter stabiler medizinischer $\mathrm{Zu}$ stand, der die entsprechenden Therapieelemente der arbeits(platz)bezogenen medizinischen Trainingstherapie („work hardening") bzw. des Arbeitssimulationstrainings („work conditioning“) zulässt. Dabei werden neben den konventionellen Elementen der physikalischen Therapie, Krankengymnastik, Ergotherapie und medizinischen Trainingstherapie auch FCE-Systeme (z. B. EFL nach Isernhagen) genutzt. Zum Abschluss der rehabilitativen Intervention sollte nochmals eine FCE-gestützte Testung des arbeits(platz) bezogenen Patientenfähigkeitsprofils vorgenommen und dieses mit dem Anforderungsprofil abgeglichen werden.

Ein möglichst realistischer Arbeitsplatzbezug während der komplextherapeutischen Rehabilitation (EAP, BGSW oder ABMR) schafft somit eine solide Grundlage für das Verständnis von Notwendigkeit und Sinn der Rehabilitationsmaßnahme für den Unfallverletzten, woraus in aller Regel eine optimale Kooperation und Motivation des Patienten („Ich trainiere genau das, was ich auf Arbeit wieder können muss") entsteht. 
Wenn die Rehabilitanden von der Sinnhaftigkeit dieser Therapie überzeugt werden können, gewinnen sie schnell verloren gegangenes Selbstvertrauen in die eigene Leistungsfähigkeit zurück und lernen, bei dauerhaft verbleibenden Verletzungsfolgen ihre Leistungsgrenzen einzuschätzen und vorhandene Defizite ergonomisch zu kompensieren. Die daraus resultierende abschließende sozialmedizinische Beurteilung der arbeits(platz)bezogenen Leistungsfähigkeit führt zur definitiven Klärung der folgenden 4 Fragen:

1. Letzte berufliche Tätigkeit uneingeschränkt weiterhin möglich?

2. Letzte berufliche Tätigkeit bei angepasstem Arbeitsplatz weiterhin möglich?

3. Berufliche Neuorientierung erforderlich?

4. Leistungsreserven erschöpft? [3]

\section{Fallkonferenz}

Die Fallkonferenz als gemeinsame Verlaufs- oder Abschlussbesprechung mit Beteiligung des Rehabilitanden, des Reha-Managers, des Reha-Mediziners und ggf. auch des behandelnden Therapeuten ist ein wichtiges und im Grunde unverzichtbares Instrument des Reha-Managements. Bei der Verlaufsfallkonferenz werden auf der Grundlage des erfolgten Eingangsassessments und unter Beachtung der Messergebnisse der PROs der Rehabilitationsverlauf beurteilt, der Rehabilitationsplan überprüft und ggf. angepasst sowie eine eventuell erforderliche Verlängerungsentscheidung unter Beachtung der mit dem Rehabilitanden abgestimmten Rehabilitationsziele getroffen. Im Rahmen der Abschlussfallkonferenz wird auf der Basis des erhobenen Abschlussassessments und der Ergebnisse der PROs geklärt, ob die eingangs gestellten Rehabilitationsziele erreicht worden sind, und nach Möglichkeit eine prognostische Aussage zum Zeitpunkt der Wiedereingliederung des Rehabilitanden in den Arbeitsprozess gemacht. Falls dies noch nicht möglich ist, wird der weitere Reha-Plan gemeinsam festgelegt, und es werden ggf. notwendige Verordnungen für Heil- und Hilfsmittel bzw. ambulante Weiterbehandlungsmaßnahmen ausgestellt und sofort vom Reha-Manager ge- nehmigt. Die während der Fallkonferenz getroffenen Vereinbarungen und Festlegungen werden in einem standardisierten Protokoll exakt dokumentiert.

Bei einigen Unfallversicherungsträgern ist die beschriebene Fallkonferenzpraxis ein obligatorisches Element des Reha-Managements. Je nach Anzahl der Reha-Managementfälle führen verschiedene Unfallversicherungsträger in regelmäßigen Abständen zu festgelegten Terminen Fallkonferenzen in unserem Haus durch. Die Mehrzahl der Reha-Träger nutzt das Instrument der Fallkonferenz nur in bestimmten Einzelfällen.

\section{Jährliches Review}

Gemeinsam mit einigen UV-Trägern führten wir als letzten wesentlichen Schritt zur Weiterentwicklung unseres Reha-Managements ein jährliches Review ein. Dabei erfolgen ein jährlicher Rückblick auf alle Reha-Fälle des Leistungsträgers und eine gemeinsame Analyse hinsichtlich der in der Abschlussfallkonferenz gestellten Prognose der Arbeitsunfähigkeitsdauer, der verbliebenen Minderung der Erwerbsfähigkeit und der angefallenen Kosten für die durchgeführten Rehabilitationsverfahren. Zudem werden bei dem Review die Verletzungsdiagnosen eingruppiert und die Messergebnisse der PROs bewertet und diskutiert sowie mit den Ergebnissen der Vorjahre verglichen. Beteiligte am Review sind (leitende) Vertreter des UV-Trägers, die für das BG-liche Heilverfahren zuständigen Reha-Mediziner der Klinik und Beratungsärzte des UV-Trägers, die im Review-Verfahren als „neutrale" Gutachter und Bewerter fungieren. Nach vorab durchgeführter Fallverlaufsanalyse durch den Unfallversicherungsträger erfolgt zum Review gemeinsam mit allen Beteiligten ein vollständiges Debriefing aller Fälle des Vorjahres. Aus den Ergebnissen werden zum Abschluss des Reviews Schlussfolgerungen hinsichtlich weiterer Optimierungsschritte und notwendiger Verfahrensänderungen getroffen. Nach mittlerweile reichlich 10-jähriger Erfahrung mit dieser Reviewpraxis lässt sich der interne Erkenntnisgewinn für die Arbeit in unserer Klinik folgendermaßen charakterisieren:
- Nachweis der Ergebnisqualität der Arbeit unserer BGSW-Klinik,

- Nachweis der Effektivität eingesetzter Reha-Methoden,

- Nachweis der Effizienz der Rehabilitation und des Reha-Managements (Heilverfahrensdauer, Kosten),

- Aussagen zu fallbezogenen Arbeitsunfähigkeitszeiten und zur Sicherheit der durch die Reha-Mediziner abgegebenen Arbeitsunfähigkeitsprognosen,

- Informationen zur fallgruppenbezogenen Verlaufsspezifik,

- Aufdeckung von Prozessschwächen und -stärken (z. B. optimaler Zeitpunkt des BGSW-Beginns bei bestimmten Verletzungen, Umsetzung von Empfehlungen; Patienten-Compliance).

Der externe Erkenntnisgewinn bei Betrachtung des Reha-Netzwerkes umfasst:

- Aussagen zur Prozessqualität des gesamten Heilverfahrens (z. B. Erkenntnisse zur Qualität und Wirksamkeit von Reha-Plänen; Erkenntnisse zu optimalen Zeitpunkten des Belastungsaufbaus bei bestimmten Verletzungen; Erkenntnisse hinsichtlich der notwendigen Kompressionstherapie in der Akutklinik, um den Reha-Prozess nicht zu verzögern),

- Aussagen zur Ergebnisqualität von Einweiserkliniken („Landkarte kompetenter Netzwerkpartner"),

- Aussagen zur Steuerungs- und RehaManagementfunktion der einweisenden D-Ärzte,

- Aussagen zum Benchmarking (Vergleich zu anderen Netzwerkkliniken).

\section{Erwartungen hinsichtlich der Weiterentwicklung des Reha-Managements der DGUV}

Im März 2013 wurde auf dem 1. DGUVForum Reha-Management in Dresden ein von den dort anwesenden Experten und Fachleuten erarbeitetes 12-Thesen-Papier vorgestellt, an dem sich die Weiterentwicklung und die praktische Umsetzung des Reha-Managements zukünftig orientieren sollen. Als eine vorrangige Aufgabe wurde dabei die weitere Optimierung von Netzwerkstrukturen, in denen die Leis- 
Infobox 3 Vorschlag für ein Phasenmodell Rehabilitation im DGUV-Heilverfahren

- Phase der Akutbehandlung (Sofortrehabilitation in Akutklinik)

- Phase der Frührehabilitation (in Akutklinik oder spezialisierter Reha-/BGSWEinrichtung oder BG-Klinik)

- Phase der postprimären Rehabilitation (in spezialisierter Reha-/BGSW-Einrichtung oder BGSW-Klinik oder BG-Klinik)

- Phase der Anschlussrehabilitation (in BGSW-Klinik oder EAP-Einrichtung oder ambulanter Therapieeinrichtung oder BG-Klinik)

- Phase der Rehabilitation zur Teilhabe (in ABMR-Einrichtung oder Berufsförderungswerk oder bei entsprechendem Bildungsträger oder als Belastungserprobung am Arbeitsplatz im Betrieb)

- Phase des Verweilzustandes, der Nachsorge oder Pflege (in D-Arzt-Praxis oder bei Erfordernis in entsprechender Pflegeeinrichtung)

tungserbringer verschiedener Fachbereiche und die Leistungsträger eng zusammen arbeiten, gesehen [14]. Daraus resultiert, dass auch die Rolle der BGSW-Kliniken in diesen Netzwerken neu definiert werden muss. Im Jahr 2006 wurde von der Deutschen Gesellschaft für Unfallchirurgie mit dem „Weißbuch“ die Schwerverletztenversorgung auf eine neue strukturelle Grundlage gestellt [15]. Kern dieser Strukturveränderung ist eine Dreistufigkeit in der Akutbehandlung Unfallverletzter mit der Bildung von Einrichtungen der Basisversorgung sowie regionalen und überregionalen Traumazentren. Bei der Neuordnung des BG-lichen Heilverfahrens entwickelte die DGUV diese Idee weiter, wobei das Modell der 3 Versorgungsstufen [Einrichtungen mit stationärem D-Arzt-Verfahren, VAV (Verletztenartenverfahren)- bzw. SAV (Schwerstverletztenartenverfahren)-Einrichtungen] dabei weitgehend übernommen wurde [16-19].

Rehabilitationskliniken können heute je nach Spezialisierungsgrad unterschieden werden in Einrichtungen der „Basisversorgung“ (Durchführung von Heilverfahren in der Phase der Anschlussrehabilitation) und Einrichtungen der „Maximalversorgung“ (Durchführung von Heilverfahren in der Phase der Früh-, postprimären und Anschlussrehabilitation sowie speziellen Heilverfahren, wie z. B. Rehabilitation von Schwerbrandverletzten). Aus unserer Sicht wäre es nun sinnvoll, dieses zweistufige Versorgungsmodell der stationären Rehabilitation in das dreistufige Versorgungsmodell im Akutbereich einzufügen und ein SGB-VII-Trauma-Reha-Netzwerk zu entwickeln. Dabei könnten BGSW-Kliniken der „Basisversorgung“ als regionale Trauma-Reha-Zentren und BGSWEinrichtungen der „Maximalversorgung“ als überregionale Trauma-Reha-Zentren fungieren und sowohl in das Trauma-Netzwerk-Konzept der DGU eingegliedert werden, als auch eine flächendeckende Versorgung der DGUV-Patienten sicherstellen. Selbstverständlich müssten seitens der DGUV hierfür die BGSW-Anforderungen und Handlungsanleitungen entsprechend überarbeitet und weiterentwickelt werden. In diesem Netzwerk muss die Anbindung geeigneter Reha-Kliniken der verschiedenen Versorgungsstufen an die SAV-, VAV- und DAV (Durchgangsarztverfahren)-Versorgungsstufen inklusive fachlicher und logistischer Vernetzung definiert und in die bereits existierende Zertifizierungssystematik eingebunden werden. Die Kliniken, die bereit sind, sich an einem solchen Netzwerk zu beteiligen und die entsprechenden Qualitäts- und Zertifizierungsanforderungen zu erfüllen, sollten dann auch bevorzugt mit DGUV-Patienten belegt werden, um ihre speziellen und hoch qualifizierten Leistungsangebote zur weiteren Verbesserung des Reha-Managements der DGUV wirkungsvoll und effizient einsetzen zu können. Daraus folgt allerdings auch eine notwendige versorgungsstufen- und leistungsorientierte Vergütungsstruktur. Die bisher mit dem Hauptbeleger der BGSW-Klinik, i. d. R. der DRV, verhandelten Kostensätze sowie der $\mathrm{Zu}$ schlag der DGUV von $25 €$ täglich für das Erfüllen weitergehender Therapieleistungen werden dem dargestellten Anspruch an "maximalversorgende" Trauma-RehaZentren nicht gerecht.

Der genannte Vorschlag zur qualitativen Etablierung der Netzwerkstruktur der DGUV sollte aus unserer Sicht zudem als Grundlage zu einer damit verknüpften Weiterentwicklung der Heilverfahrens- struktur und -steuerung genutzt werden. Die bisher existierenden komplextherapeutischen Rehabilitationsverfahren (KSR, BGSW, EAP, ABMR, spezielle Heilverfahren) stehen im Reha-Management der DGUV als Bausteine aus einem Leistungskorb zur Verfügung, die zu geeigneten Zeitpunkten und bei definiertem Bedarf der Unfallverletzten zur Anwendung kommen können. Aus unserer Sicht muss die zukünftige Weiterentwicklung des Heilverfahrens der DGUV hin zu einem Phasenmodell erfolgen, in dem die jeweils geeigneten Heilverfahrensbausteine einzuordnen sind. Innerhalb des Modells kann der Rehabilitationsprozess grundsätzlich in eine Abfolge von Phasen untergliedert werden, die von den Rehabilitanden je nach Verletzungsart und -schwere vollständig oder partiell durchlaufen werden (vgl. auch [20]; - Infobox 3).

Bei Betrachtung der aktuellen Situation zeigt sich, dass für die Phasen der Frührehabilitation und der postprimären Rehabilitation bislang noch keine spezifischen Rehabilitationsverfahren der DGUV existieren. Die Indikation zur „klassischen“ BGSW wird entsprechend den aktuellen Handlungsanleitungen nach wie vor an den Prinzipien der Rehabilitationsfähigkeit der Deutschen Rentenversicherung festgemacht, was zur Folge hat, dass Patienten dieser frühen Reha-Phasen nach Abschluss der Akutversorgung bis zum Erreichen der Rehabilitationsfähigkeit in ein „Reha-Loch“ fallen. In der aktuellen Praxis kann es deshalb dazu kommen, dass diese Fälle entweder in Pflegeeinrichtungen oder betreuten häuslichen Situationen „Zwischengeparkt“ werden oder zu früh in eine BGSW-Klinik verlegt werden, die dann rehabilitative Leistungen erbringen muss, welche oftmals das in den BGSWStandards geforderte und durch entsprechende Pflegesätze abgesicherte Leistungsportfolio bei Weitem übersteigen. In einigen Fällen werden derartige Fälle aber auch auf der Basis von Einzelfallentscheidungen der Unfallversicherungsträger in entsprechend qualifizierte und in den Phasen der Früh- und postprimären Rehabilitation erfahrene Häuser eingewiesen, um einen lückenlosen Reha-Prozess im Rahmen des individuellen Reha- 
Managements zu gewährleisten, wobei sich die Pflegesätze dann meist an dem Phasenmodell der neurologischen Rehabilitation orientieren. Eine Weiterentwicklung des Heilverfahrens und des Reha-Managements der DGUV, z. B. in Anlehnung an das erfolgreiche Phasenmodell neurologische Rehabilitation (VDR/ BAR 1995), wäre zur Vereinfachung der täglichen Praxis im Umgang mit derartigen Fällen, verbunden mit einer leistungsgerechten Vergütung, dringend geboten.

Ein eigener Vorschlag zur Klärung der Problematik der Rehabilitationsfähigkeit, der auch Eingang in das Weißbuch Schwerverletztenversorgung gefunden hat, orientiert darauf, dass der Zeitpunkt der Verlegung des Patienten von der Akut- in die Rehabilitationseinrichtung von der Verletzungsart, der stattgehabten operativen Behandlung, von fallbezogenen internen und externen Kontextfaktoren sowie vom avisierten Teilhabeziel abhängt. Die Rehabilitationseinrichtung muss hinsichtlich ihrer personellen, räumlichen sowie technisch-apparativen Ausstattung in der Lage sein, für den Patienten situationsgerecht eine optimale rehabilitative Behandlung leisten zu können [15].

Ein weiterer notwendiger Entwicklungsschritt bei der Fortentwicklung des Reha-Managements der DGUV ist aus Sicht der Reha-Kliniken die Entwicklung einheitlicher Standards in der Umsetzung des Reha-Managements bei allen Unfallversicherungsträgern unter Beachtung der branchenspezifischen Besonderheiten, sodass ein trägerübergreifendes Trauma-Reha-Netzwerk entstehen kann.

Bei der Weiterentwicklung der Handlungsanleitung für die BGSW sollte neben den bereits angeführten Sachverhalten auch berücksichtigt werden, dass in der aktuellen Version die medizinischen Gründe zur Einleitung einer BGSW zu restriktiv formuliert sind und bei strenger Auslegung fast nur für Rehabilitanden in den Phasen der Früh- bzw. postprimären Rehabilitation gegeben wären. Auch die Vorgabe, wonach in der Regel keine Indikation für BGSW bei Monoverletzungen besteht, bedarf einer Überarbeitung, da sie nicht der aktuellen Reha-Managementpraxis entspricht und den Aspekt der oftmals begleitenden und wichtigen Kontextfaktoren durch die notwendige Einzelfallentscheidung nach Ziffer 3.2.2 der Handlungsanleitung BGSW nicht ausreichend einbezieht.

Resultierend aus den eingangs dargestellten eigenen Erfahrungen sind unsere Erwartungen hinsichtlich der Weiterentwicklung des Reha-Managements der DGUV insbesondere darauf gerichtet, dass zukünftig der Arbeitsplatzbezug und die Nutzung des ABMR-Verfahrens noch stärker in den Fokus genommen werden als bisher. Der systematische Einsatz von patientenbezogenen Assessmentinstrumenten sowie die Instrumente der Fallkonferenz und des Reviews sollten als Standardinventar einer qualifizierten Trauma-Reha-Netzwerk-Klinik entwickelt werden. Zudem wird es in der $\mathrm{Zu}$ kunft eine wesentliche Aufgabe aller am Reha-Management beteiligten Akteure sein, in Zusammenarbeit mit Universitäten und Forschungseinrichtungen wissenschaftlich fundierte Nachweise zur Effektivität und Kosteneffizienz des Reha-Managements der DGUV zu erbringen.

\section{Fazit für die Praxis}

- Rehabilitations- bzw. BGSW-Kliniken können als Netzwerkpartner im Heilverfahren der DGUV gemeinsam mit D-Ärzten und Reha-Managern eine Schrittmacher- und Lotsenfunktion übernehmen.

- Eigene Erfahrungen zeigen, dass der Einsatz patientenbezogener Assessment instrumente, die Nutzung arbeitsplatzbezogener Test- und Therapieelemente, die Durchführung regelhafter Fallkonferenzen und jährliche Reviews hinsichtlich der geleisteten Arbeit und erreichten Reha-Ergebnisse entscheidende Kernpunkte zur Optimierung des Reha-Managements darstellen.

- PROs in den Bereichen allgemeine und spezifische Gesundheit sowie Lebensqualität helfen bei der Aufdeckung von larvierten Kontextfaktoren, können für die individuelle RehaSteuerung genutzt werden, dienen der laufenden Effektivitätskontrolle sowie der stufenweisen Prozessoptimierung der Heilverfahren und kön- nen Grundlage für Effektivitäts- und Kosteneffizienzanalyseverfahren sein. - Die Implementierung von arbeits(platz)bezogenen Rehabilitationskonzepten auf der Basis strukturierter Vergleiche von Arbeitsplatzanforderungs- und Patientenfähigkeitsprofilen kann Rehabilitationsergebnisse signifikant verbessern und gehört zu den Kernkompetenzen moderner BGSW-Kliniken.

- Der Ausbau der von Leistungsträgern und Leistungserbringern geprägten DGUV-Netzwerkstruktur hin zu einem Trauma-Reha-Netzwerk mit Einbindung entsprechend qualifizierter Rehabilitationskliniken ist zur weiteren Optimierung des Reha-Managements der DGUV geboten.

- Die Entwicklung einheitlicher Standards in der Umsetzung des Reha-Managements bei allen Unfallversicherungsträgern unter Beachtung branchenspezifischer Besonderheiten hin zu einem trägerübergreifenden Trauma-Reha-Netzwerk ist notwendig.

- Durch die Etablierung eines Phasenmodells der Rehabilitation im Heilverfahren der DGUV könnten aktuell noch bestehende Heilverfahrensversorgungslücken geschlossen werden.

- Wissenschaftlich fundierte Nachweise zur Effektivität und Kosteneffizienz des Reha-Managements der DGUV sind in der Zukunft unverzichtbar.

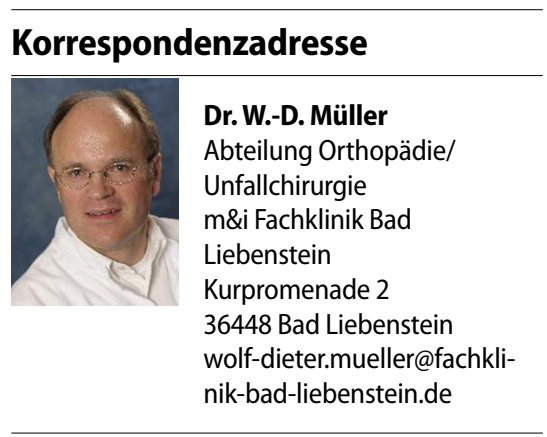

Einhaltung ethischer Richtlinien

Interessenkonflikt. W.-D. Müller gibt an, dass kein Interessenkonflikt besteht.

Dieser Beitrag beinhaltet keine Studien an Menschen oder Tieren.

The supplement containing this article ist not sponsored by industry. 


\section{Literatur}

1. DGUV Spitzenverband (2008) Eckpunkte für ein gemeinsames Reha-Management in der gesetzlichen Unfallversicherung, Stand 25.02.2008

2. DGUV Spitzenverband (2010) Das Reha-Management der Deutschen Gesetzlichen Unfallversicherung - Handlungsleitfaden vom 13.09.2010 in der Fassung vom 17.07.2014

3. Müller W-D, Lohsträter A et al (2008) Optimierung der Berufsgenossenschaftlichen Stationären Weiterbehandlung (BGSW) durch systematischen Einsatz von Assessmentinstrumenten. Phys Med Rehab Kuror 18:270-278

4. Müller W-D, Kropf U et al (2010) Erfolgsfaktoren für die Rehabilitation. Trauma Berufskrankh 12(Suppl 2):208-215

5. Bullinger M, Kirchberger I (1998) SF - 36 Fragebogen zum Gesundheitszustand - Manual. Hogrefe, Göttingen

6. Germann G, Harth A et al (2002) DASH Deutsche Version, Klinik für Hand-, Plastische und Rekonstruktive Chirurgie - Schwerbrandverletztenzentrum - BG-Unfallklinik Ludwigshafen, Klinik für Plastische und Handchirurgie der Universität Heidelberg

7. Kohlmann T, Raspe HH (1996) Der Funktionsfragebogen Hannover zur alltagsnahen Diagnostik der Funktionsbeeinträchtigungen durch Rückenschmerzen (FFbH-R). Rehabilitation 35:I-VIII

8. Stucki G, Meier D et al (1996) Evaluation einer deutschen Version des WOMAC (Western Ontario and McMaster Universities Arthroseindex). Z Rheumatol 55:40-49

9. Rabin R, de Charro F (2001) EQ5D: A measure of health status from the EuroQol Group. Ann Med 33(5):337-343

10. Maier V, Müller W-D et al (2001) Verzahnung zwischen medizinischer Rehabilitation und beruflicher Reintegration - Kosteneffektivitäts- und Kosten-Nutzwert-Analyse. Phys Med Rehab Kuror 11:145

11. Müller W-D, Maier V et al (2006) Verzahnung zwischen medizinischer Rehabilitation und beruflicher Reintegration bei Beschäftigten der Automobilindustrie mit Rücken- und Gelenkschmerzen. Phys Med Rehab Kuror 16:149-154

12. Müller W-D, Bak P et al (2005) Ergebnisorientierte Optimierung der Rehabilitationskonzepte bei berufstätigen Patienten mit rezidivierenden Rückenschmerzen - eine kontrollierte klinische Studie, 14. Rehabilitationswissenschaftliches Kolloquium, 2005. DRV-Schriften, Bd 59. Hannover, Frankfurt a. M., S 258-259

13. IMBA (2000) [Integration von Menschen mit Behinderungen in die Arbeitswelt] Einführung, das Instrument für Fachkräfte der beruflichen Rehabilitation und Integration, Papierversion, Essen IMBATeam

14. Habekost D, Kaiser L (2013) 1. DGUV-Forum RehaManagement, Ergebnisse und Perspektiven. DGUV-Forum 11:10-11

15. Weißbuch Schwerverletztenversorgung (2015) der Deutschen Gesellschaft für Unfallchirurgie, 2. Aufl. http://www.dgu-online.de/qualitaet-sicherheit/ schwerverletzte/weissbuch-schwerverletztenversorgung.html. (Stand 01/2015)

16. Bühren V (2010) Heilverfahren der DGUV. Trauma Berufskrankh 12:153-156

17. Oberscheven M (2013) Neuausrichtung der stationären Heilverfahren. Trauma Berufskrankh 15:2528
18. Oberscheven M, Kranig A (2014) Neurausrichtung der stationären Heilverfahren. Trauma Berufskrankh 16:5-8

19. Auhuber TC, Reimertz C et al (2015) Neuausrichtung der Heilverfahren der Gesetzlichen Unfallversicherung. Orthop Unfallchir up2date 10:51-69

20. Oberscheven M (2012) Neuausrichtung der stationären Heilverfahren. Trauma Berufskrankh 14:6163 